\title{
Computational Study on Characteristic Radiation Originated from Channeled Relativistic Electrons in Single-Wall Carbon Nanotubes
}

\author{
Marwa H. Ali \\ Physics department, Faculty of Science, Suez-Canal University, Ismailia 41522, Egypt
}

\begin{tabular}{|c|c|}
\hline ARTICLE INFO & ABSTRACT \\
\hline & \multirow{7}{*}{$\begin{array}{l}\text { Calculation of the number of bound states } n \text { for channeled electrons through single } \\
\text { wall carbon nanotubes }(\mathrm{n}, \mathrm{m}) \text { at different values of electrons energy by using (Wetzel, } \\
\text { Kramer's, Brillouin approximation)(WKB) method. The calculations executed according } \\
\text { to the continuum model approximation given by Lindhard for the case was carried out of } \\
\text { an axial channeling in single crystals. The estimated results of the maximum number of } \\
\text { bound states of the channeled electrons in a zigzag (n, o), armchair (n,n) and chiral(n,m) } \\
\text { nanotubes have been performed using the Moliere potential. In this work we determined } \\
\text { the emitted photon energy due to } n,(n-1) \text { transitions between higher -quantum states as a } \\
\text { function of the electron energy up to } 500 \mathrm{MeV} \text {. Also the energy levels of electrons } \\
\text { channeled in different types of single wall carbon nanotubes by using Moliere potential } \\
\text { were defined. }\end{array}$} \\
\hline Received: $19^{\text {th }}$ June 2020 & \\
\hline Accepted: $1^{\text {st }}$ Apr. 2021 & \\
\hline Keywords: & \\
\hline Channeling radiation, & \\
\hline $\begin{array}{l}\text { Single-walled carbon } \\
\text { nanotubes, }\end{array}$ & \\
\hline \multirow[t]{2}{*}{ Bound states. } & \\
\hline & $\begin{array}{l}\text { It has been showed that the emitted photon energy in the forward direction is the } \\
\text { energy difference between the successive initial and final states of the channeled electron. } \\
\text { The energy of the emitted channeling radiation has been calculated for incident electron } \\
\text { at } 50 \mathrm{MeV} \text {. We calculated the emitted channeling radiation for incident electrons at } 10 \text {, } \\
50 \text { and } 500 \mathrm{MeV} \text { with frequency in X-ray range. }\end{array}$ \\
\hline
\end{tabular}

\section{1-INTRODUCTION}

In previous work, the channeling of negatively charged particles in disordered lattices of cubic crystals including the characteristics of channeling radiation that emits spontaneously of the channeled electrons [1] in addition to the calculations of the transmission and dechanneling coefficients in disordered lattices was considered [2]. The first theoretical study of particle channeling in CNTs showed those relativistic positrons and electrons can emit, respectively, quasimonochromatic hard X-rays and $\gamma$-rays when channeled in CNTs. Although the creation and transport of highenergy electromagnetic radiation represents an important line of research in the area of particle interactions with CNTs[3] .This radiation was predicted by Kumakhov [4, 5] and is called now channeling radiation. Also in previous calculations, the eigenvalues of the bound states of the channeled particles have been obtained in harmonic and in a harmonic approximation of the planar potential have been performed using the first-order perturbation theory [6]. It has been found that, in the harmonic approximation, the allowed transitions occur at
$\Delta \mathrm{n}= \pm 1$, while in the case of a harmonic up to $\mathrm{x} 6$ term, the allowed transitions occur at $\Delta \mathrm{n}= \pm 1, \pm 3, \pm 5$.

In this work, the calculations of the bound states of the channeled particles are executed by using the WKB approximation method, It has been found that, the allowed transitions occur at $\Delta \mathrm{n}= \pm 1$.

The emitted photon energy in the forward direction is given by $h \omega=2 \gamma^{2}(\Delta \mathrm{E})$ where $\Delta \mathrm{E}=\mathrm{E}_{\mathrm{n}+1}-\mathrm{E}_{\mathrm{n}}$ is the energy difference between the successive initial and final states of the channeled electrons [7]. One of the most important applications of carbon nanotubes is the channeling of charged particles through CNTs. The first theoretical study of particle channeling in CNTs appeared in the work of Klimov and Letokhov [8], have suggested relativistic positrons and electrons can emit, respectively monochromatic hard X-rays and gammarays when channeled in CNTs. In the work of Dedkov[9] gave qualitative arguments showing that CNTs should provide more favorable conditions for ion channeling than single crystals[10]. 


\section{Radiation Characteristics of Channeled Electrons.}

In previous work [11], the nanotube channeling potential has been calculated according to the continuum model approximation given by Lindhard [1], and by using the atomic interaction potential as given by Moliere [12], Classical channeling and channeling radiation in a nanotube was first considered by Klimov and Letokhov, their calculations were based on the continuum potential [3].

\section{CHANNELING POTENTIAL IN CARBON NANOTUBES}

According to the continuum model approximation given by Lindhard [1] for the case of an axial channeling in single crystals, called continuum potential. The continuum potential of single atomic row can be written as:

$$
\mathrm{V}(\mathrm{r})=\frac{1}{\mathrm{~d}_{\mathrm{R}}} \int_{-\infty}^{\infty} \mathrm{V}\left(\sqrt{\mathrm{r}^{2}+\mathrm{z}^{2}}\right) \mathrm{dz}
$$

In this work [9], the calculations of potential are executed by using the atomic interaction potential as is given by Moliere potential as [10]:

$$
V_{M}(r)=\frac{z_{1} z_{2} e^{2}}{r} \sum_{i=1}^{3} \alpha_{i} \exp \left(-\beta_{i} r\right)
$$

,with $\left\{\alpha_{i}\right\}=\{0.35,0.55,0.1\},\left\{\beta_{i}\right\}=\{0.3 / a, 1.2 / a, 6 / a\}$ where $\mathrm{a}=\left(9 \pi^{2} / 128 \mathrm{z}_{2}\right)^{1 / 3} \mathrm{a}_{0}$, is the Thomas-Fermi screening radius; $a_{0}=0.529 \AA$ is the Bohr radius, $\mathrm{z}_{1}$ and $\mathrm{z}_{2}$ are the charge numbers of projectile and target atoms, respectively, $\mathrm{e}$ is the elementary charge and $\mathrm{r}$ is the separation between them.

Using the above expressions given by Eq. (2) into Eq. (1), we can obtain the axial potential corresponding to Moliere potential as the following:

$$
\mathrm{V}_{\mathrm{M}}(\mathrm{r})=\frac{2 \mathrm{z}_{1} \mathrm{z}_{2} \mathrm{e}^{2}}{\mathrm{~d}_{\mathrm{R}}} \sum_{\mathrm{i}=1}^{3} \alpha_{\mathrm{i}} \mathrm{K}_{0}\left(\beta_{\mathrm{i}} \mathrm{r}\right)
$$

\section{DERIVATION OF EQUATIONS}

Calculation of the energy eigenvalues, and the maximum number of bound states, from ref. [10], and using the WKB method [3], the energy eigenvalues and the maximum number of bound states respectively were gotten as:

$$
\mathrm{E}_{\mathrm{n}}=-\mathrm{a}_{1}+\left(\frac{1}{2 \mathrm{~m}_{0} \gamma}\right)\left(\frac{\mathrm{c}_{1} \pi \mathrm{h}}{2 \ln 2}\right)^{2}\left(\mathrm{n}+\frac{1}{2}\right)^{2}
$$

, and

$$
\mathrm{n}_{\max }=\left(\frac{2 \ln 2}{c_{1} \pi \mathrm{h}}\right)\left[2 \mathrm{~m}_{0} \gamma\left(\mathrm{a}_{1}-\mathrm{E}_{\max }\right)\right]^{1 / 2}-0.5
$$

,where $\mathrm{E}_{\max }$, is the potential at the turning points, that is $\mathrm{E}_{\max }=\mathrm{a}_{1}+\mathrm{b}_{1} \mathrm{e}^{-\mathrm{c}_{1} \mathrm{~s}}, s=R-a$, is the screening length, $\mathrm{R}=\mathrm{d} / 2$ being the nanotube radius and $\mathrm{a}=\left(9 \pi^{2} / 128 \mathrm{z}_{2}\right)^{1 / 3} \mathrm{a}_{0}$, is the Thomas-Fermi screening radius; $\mathrm{a}_{0}=0.529 \AA$ is the Bohr radius, $\mathrm{z}_{2}$ is the charge number of the target atoms, and $\mathrm{e}$ is the elementary charge.

The energy eigenvalues of relativistic electrons channeled through single-wall carbon nanotubes could be used in the calculations of the energy of the emitted channeling radiation. The emitted photon energy in the forward direction is given by [7]:

$$
\mathrm{h} \omega=2 \gamma^{2}(\Delta \mathrm{E})
$$

, where $\Delta \mathrm{E}=\mathrm{E}_{\mathrm{n}+1}-\mathrm{E}_{\mathrm{n}}$ is the energy difference between the successive initial and final states of the channeled electrons.

Calculation of the wave length of the emitted radiation

The wave length of the emitted radiation $\lambda$, is calculated by using Eq. (6) as follows:

$$
\lambda=\frac{\mathrm{ch}}{2 \gamma^{2}(\Delta \mathrm{E})}
$$

, where, $\mathrm{c} \approx 3 \times 10^{10} \mathrm{~cm} / \mathrm{sec}$ is the speed of light.

\section{RESULTS AND DISCUSSION.}

\subsection{Calculation The maximum number of bound states $\left(\mathbf{n}_{\text {max }}\right)$ :}

The maximum of the number of bound states $n_{\max }$ calculated by using WKB approximation method as calculated from Eq. (5) at different values of electron energy $(10,15,20,25,50,100,200$, and $500 \mathrm{MeV})$ are given for electron channeled in single wall carbon nanotubes (armchair, Chiral ,and Zigzag) was given in Table $(1,2$,and 3$)$ respectively. 
The calculations show that the estimation of the maximum number of bound states of the channeled electron for both armchair chiral, and zigzag nanotube at low incident energy are found to be $\mathrm{n}_{\max }$ approximately equal. While at high incident energy are found to be $\mathrm{n}_{\max }$ for armchair type $\mathrm{n}_{\max }(10,10)>\mathrm{n}_{\max }(8,8)>\mathrm{n}_{\max }$ $(5,5)$, also $n_{\max }$ for Chiral $n_{\max }(15,6)>n_{\max }(16,4)>n_{\max }$ $(11,9) \mathrm{n}_{\max }(11,5)>\mathrm{n}_{\max }(8,2)$, and $\mathrm{n}_{\max }$ for zigzag $\mathrm{n}_{\max }$ $(18,0)>n_{\max }(10,0)>n_{\max }(8,0) n_{\max }(6,0)$ these results shows that the maximum number of bound states of the channeled electron for both armchair chiral, and zigzag nanotube at high incident energy are increase with increasing of single wall carbon nanotubes radii and ,the relation between the maximum number of bound states, $\mathrm{n}_{\max }$ of the channeled electrons as a function of the nanotube radius at different incident energies (50, 100, and 500) $\mathrm{MeV}$ are shown in Figs. 1, 2, and 3 respectively.

\subsection{Study of the emitted photon energy due to the allowed transitions $n, n-1$ for electrons with incident (low, medium, and high) energy.}

Study the emitted photon energy due to the allowed transitions $n, n-1$ for electrons with incident low energy $E=10 \mathrm{MeV}$ channeled in armchair single-walled carbon nanotube selected with different diameters as shown in Fig. 4,study the emitted photon energy due to the allowed transitions $n, n-1$ for electrons with incident medium energy $E=50 \mathrm{MeV}$ channeled in zigzag singlewalled carbon nanotube selected with different diameters shows that in Fig. 5,,and study the emitted photon energy due to the allowed transitions $n, n-1$ for electrons with incident high energy $E=500 \mathrm{MeV}$ channeled in chiral single-walled carbon nanotube selected with different diameters shows that in Fig. 6.

\subsection{Calculation the wave length of the emitted radiation $\lambda$ at different incident electron energy.}

The wave length of the emitted radiation $\lambda$ at different incident energy as calculated from Eq. (7).The relation between the radius of (zigzag, chiral, armchair) single wall carbon nanotubes and emitted radiation wavelength at different incident energy (50, 100, and 500) $\mathrm{MeV}$ as shown in Fig. (7, 8, and 9) respectively. The calculations results showed the emitted radiation energy range lies in the X-ray band, also with increasing the incident electron energy the emitted radiation energy increase towards the $\gamma$ - energy band and accordingly, the wave length decrease towards the $\gamma$-band. For all values of electron energy, the wave length of the emitted radiation was found to be a decreasing function of the nanotube radius while the maximum number of bound states of the channeled electrons is an increasing function of the nanotube radius for the different types of SWCNTs namely, zigzag, armchair and chiral.

Table (1): The maximum number of bound states, $\mathbf{n}_{\max }$ for channeling electrons in single wall carbon nanotubes (armchair) at different values for electron energy by using WKB approximation

\begin{tabular}{lcccccccc}
\hline \multicolumn{1}{c}{ electron energy $(\mathbf{M e V})$} & $\mathbf{1 0}$ & $\mathbf{1 5}$ & $\mathbf{2 0}$ & $\mathbf{2 5}$ & $\mathbf{5 0}$ & $\mathbf{1 0 0}$ & $\mathbf{2 0 0}$ & $\mathbf{5 0 0}$ \\
\hline $\begin{array}{l}\mathbf{n}_{\max } \text { for }(\mathbf{5 , 5}) \\
(\mathbf{R}=\mathbf{0 . 3 3 6 6 1 3} \mathbf{~ n m})\end{array}$ & 4 & 5 & 6 & 7 & 10 & 14 & 20 & 32 \\
$\begin{array}{l}\mathbf{n}_{\max } \text { for }(\mathbf{8}, \mathbf{8}) \\
(\mathbf{R}=\mathbf{0 . 5 3 8 5 8} \mathbf{~ n m})\end{array}$ & 5 & 6 & 7 & 7 & 11 & 15 & 22 & 35 \\
$\begin{array}{l}\mathbf{n}_{\max } \text { for }(\mathbf{1 0 , 1 0}) \\
(\mathbf{R}=\mathbf{0 . 6 7 3 2 2 5} \mathbf{~ n m})\end{array}$ & 5 & 6 & 7 & 8 & 12 & 17 & 24 & 37 \\
\hline
\end{tabular}

Table (2): The maximum number of bound states, $\mathbf{n}_{\max }$ for channeling electrons in single wall carbon nanotubes (Chiral) different values for electron energy by using WKB approximation

\begin{tabular}{|c|c|c|c|c|c|c|c|c|}
\hline electron energy $(\mathrm{MeV})$ & 10 & 15 & 20 & 25 & 50 & 100 & 200 & 500 \\
\hline $\begin{array}{l}n_{\max } \operatorname{for}(8,2) \\
(R=0.356237 \mathrm{~nm})\end{array}$ & 3 & 4 & 4 & 5 & 7 & 10 & 14 & 22 \\
\hline $\begin{array}{l}n_{\max } \operatorname{for}(11,5) \\
(R=0.551059 \mathrm{~nm})\end{array}$ & 4 & 4 & 5 & 6 & 9 & 12 & 17 & 34 \\
\hline $\begin{array}{l}n_{\max } \text { for }(11,9) \\
(R=0.674347 \mathrm{~nm})\end{array}$ & 6 & 7 & 8 & 9 & 13 & 19 & 27 & 43 \\
\hline $\begin{array}{l}n_{\max } \text { for }(16,4) \\
(R=0.712475 \mathrm{~nm})\end{array}$ & 6 & 7 & 9 & 10 & 14 & 20 & 28 & 44 \\
\hline $\begin{array}{l}n_{\max } \text { for }(15,6) \\
(R=0.728205 \mathrm{~nm})\end{array}$ & 6 & 8 & 9 & 13 & 14 & 20 & 28 & 45 \\
\hline
\end{tabular}


Table (3): The maximum number of bound states, $\mathbf{n}_{\max }$ for channeling electrons in single wall carbon nanotubes (Zigzag) at different values for electron energy by using WKB approximation

\begin{tabular}{|c|c|c|c|c|c|c|c|c|}
\hline electron energy $(\mathrm{MeV})$ & 10 & 15 & 20 & 25 & 50 & 100 & 200 & 500 \\
\hline $\begin{array}{l}\mathrm{n}_{\max } \text { for }(6,0) \\
(\mathrm{R}=0.233212 \mathrm{~nm})\end{array}$ & 2 & 2 & 3 & 3 & 5 & 7 & 10 & 16 \\
\hline $\begin{array}{l}n_{\max } \operatorname{for}(8,0) \\
(R=0.31095 \mathrm{~nm})\end{array}$ & 2 & 3 & 3 & 4 & 6 & 8 & 12 & 19 \\
\hline $\begin{array}{l}n_{\max } \text { for }(10,0) \\
(R=0.3887 \mathrm{~nm})\end{array}$ & 2 & 3 & 4 & 4 & 6 & 8 & 12 & 19 \\
\hline $\begin{array}{l}n_{\max } \operatorname{for}(18,0) \\
(R=0.699636 \mathrm{~nm})\end{array}$ & 5 & 7 & 8 & 9 & 12 & 17 & 25 & 40 \\
\hline
\end{tabular}

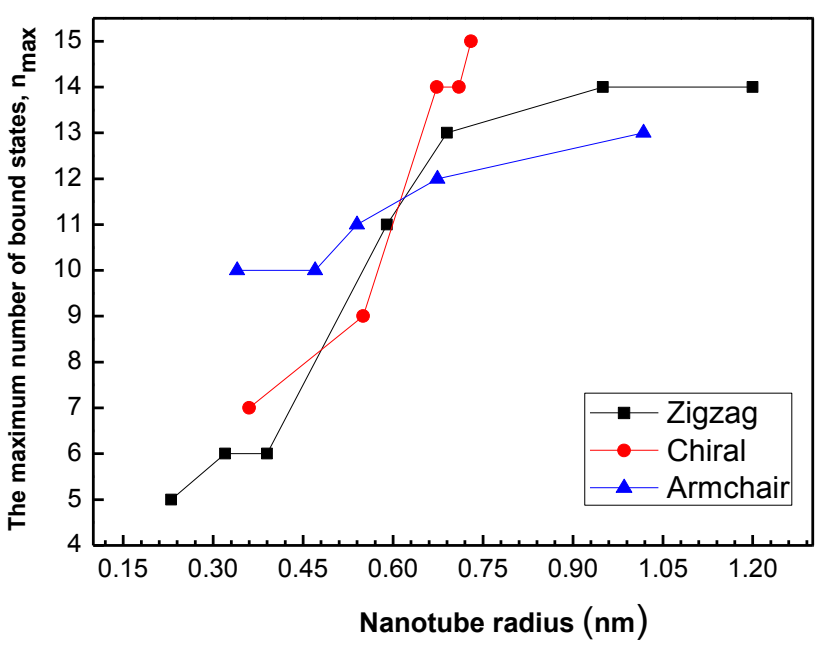

Fig. (1): The relation between the radius and the maximum number of bound state, $n_{\max }$ in (zigzag, chiral, armchair) single wall carbon nanotubes at $50 \mathrm{MeV}$.

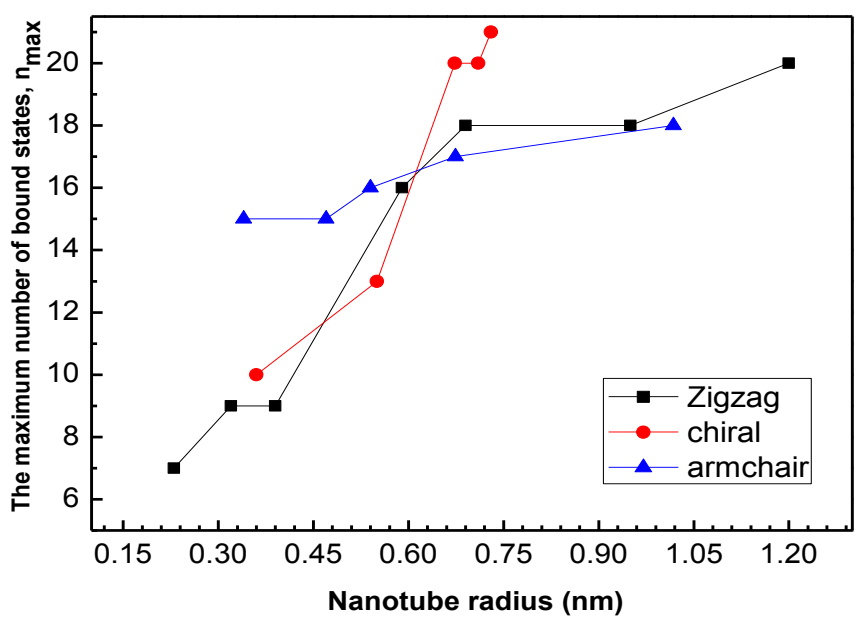

Fig. (2): The relation between the radius and the maximum number of bound state, $\mathbf{n}_{\max }$ in (zigzag, chiral, armchair) single wall carbon nanotubes at $100 \mathrm{MeV}$.

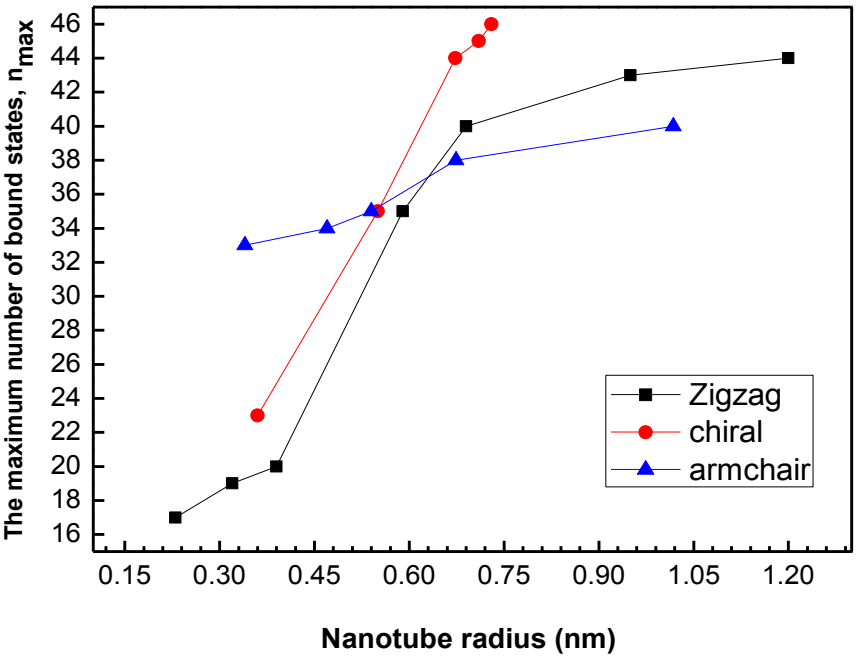

Fig. (3): The relation between the radius and the maximum number of bound state, $\mathbf{n}_{\max }$ in (zigzag, chiral, armchair) single wall carbon nanotubes at $500 \mathrm{MeV}$.

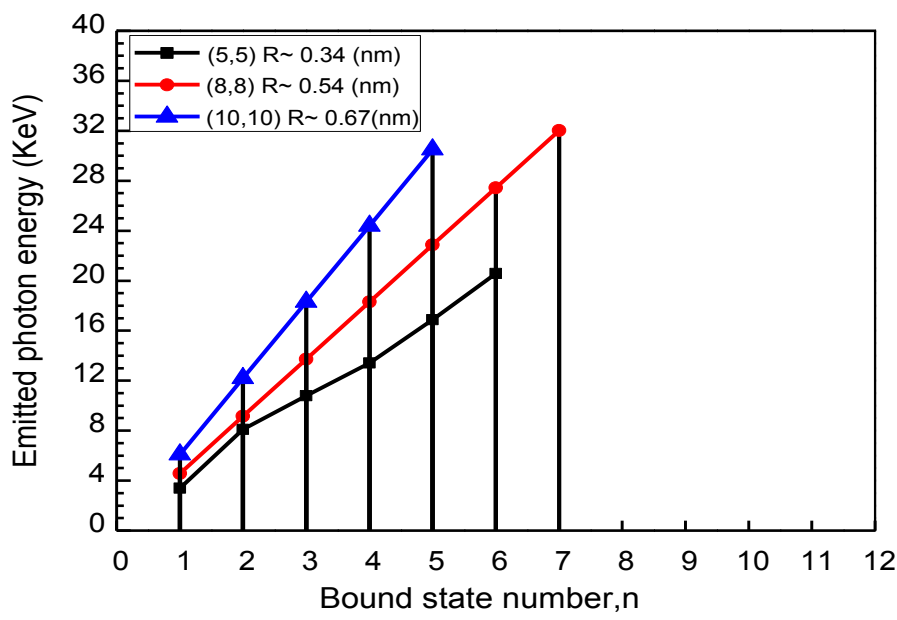

Fig. (4): The emitted photon energy due to the allowed transitions $n, n-1$ for electrons with $E=10$ MeVchanneled in armchair single-walled carbon nanotube selected with different diameters. 


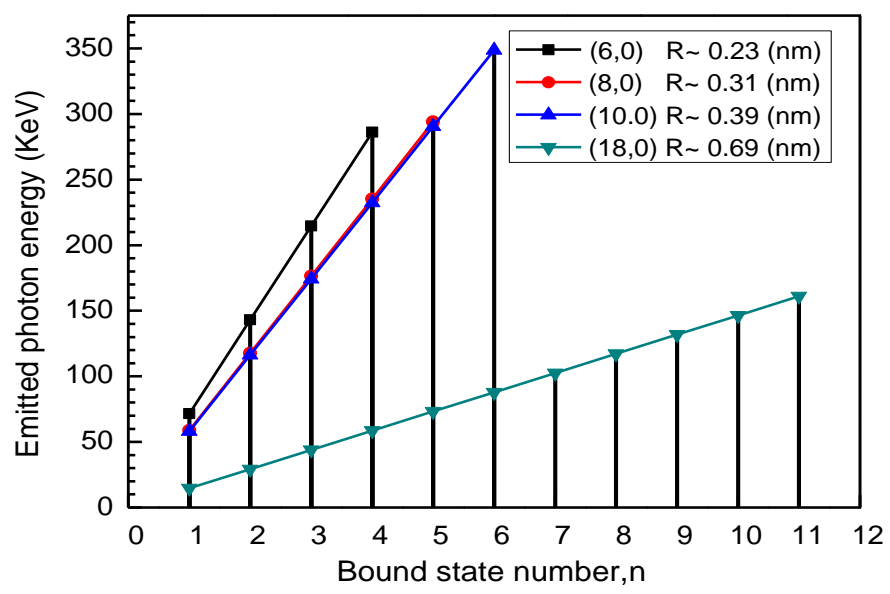

Fig. (5): The emitted photon energy due to the allowed transitions $n, n-1$ for electrons with $E=50 \mathrm{MeV}$ channeled in zigzag single-walled carbon nanotube selected with different diameters.

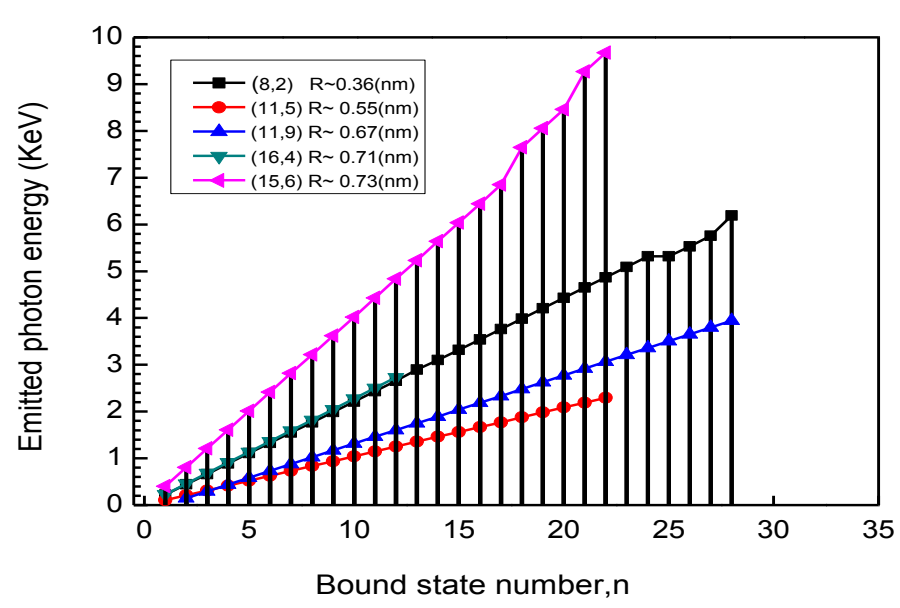

Fig. (6): The emitted photon energy due to the allowed transitions $n, n-1$ for electrons with $E=500 \mathrm{MeV}$ channeled in chiral single-walled carbon nanotube selected with different diameters.

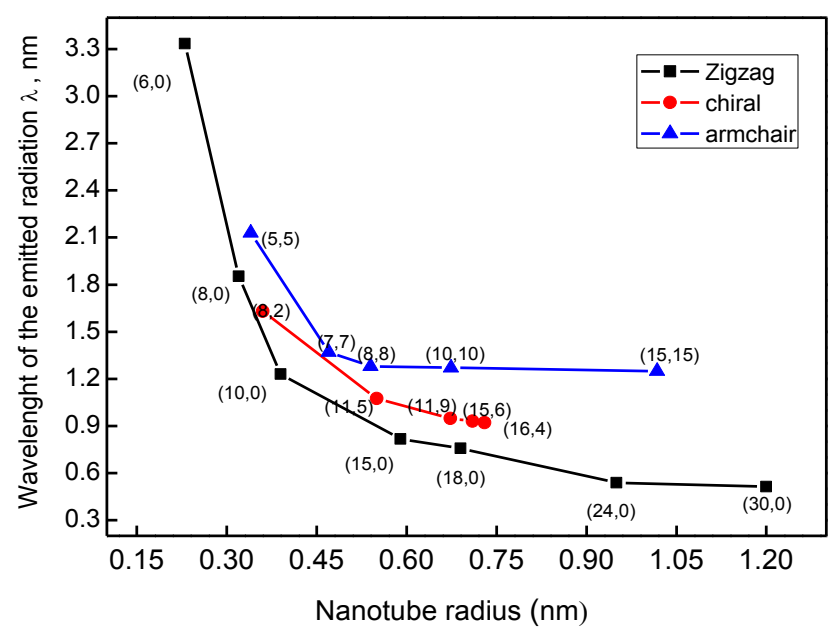

Fig. (7): The relation between the radius and wavelength in (zigzag, chiral, armchair) single wall carbon nanotubes at $50 \mathrm{MeV}$.

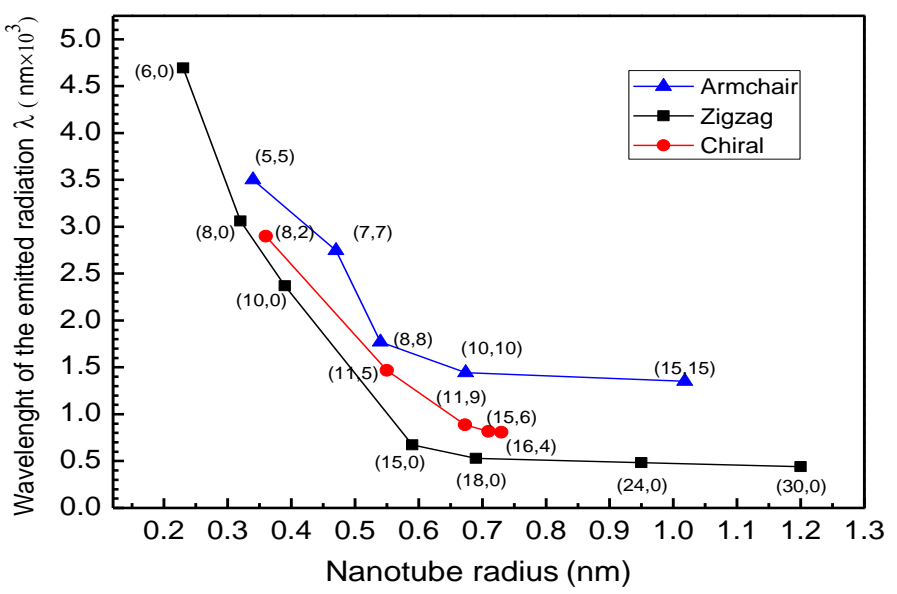

Fig. (8): The relation between the radius and wavelength in (zigzag, chiral, armchair) single wall carbon nanotubes at $100 \mathrm{MeV}$.

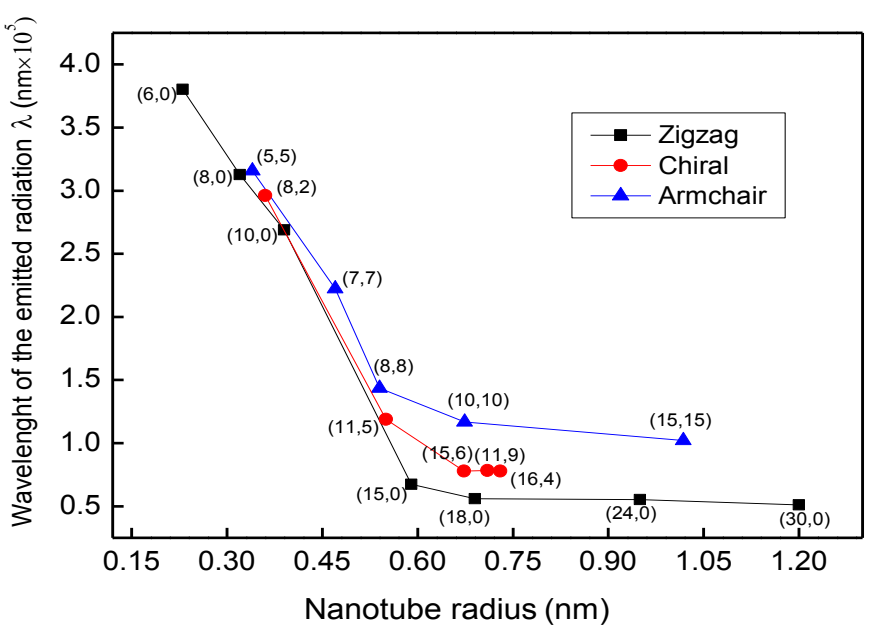

Fig. (9): The relation between the radius and wavelength in (zigzag, chiral, armchair) single wall carbon nanotubes at $500 \mathrm{MeV}$.

\section{CONCLUSIONS}

In this study, the energy eigenvalues were calculated. Determination of the maximum number of bound states nmax, are given for electron channeled in single wall carbon nanotubes (armchair, Chiral, and Zigzag) at different values of electron energy $(10,15,20,25,50,100,200$, and $500 \mathrm{MeV})$. Calculated the energy of the emitted channeling radiation for incident electron at $50 \mathrm{MeV}$. The emitted channeling radiation for incident electrons at 10,50 and $500 \mathrm{MeV}$ with frequency in X-ray range. The emitted channeling radiation for incident electron at 10,50 and $500 \mathrm{MeV}$ with wavelength in $(\mathrm{nm})$. The relation between the radius and the maximum number of bound state, $\mathrm{n}_{\max }$ in (zigzag, chiral, armchair) single wall carbon nanotubes at $(50,100$ ,and500MeV). 


\section{REFERENCE}

[1] J. Lindhard, Influence of crystal lattice on motion of energetic charged particle, Mat. Fys. Medd. Dan. Vid. Selsk. 34 (14) (1965).

[2] M.K. Abu-Assy, Effect of temperature on transmission of planar channeled positrons in cubic metals containing point defects, Nuclear Instruments \& Methods in Physics Research Section B-Beam Interactions with Materials and Atoms, 267 (2009) 2515-2520.

[3] Z. L. Miskovic, Radiation Effects \& Defects in Solids, Vol.162, Nos. 3-4, March-April (2007) 185205.

[4] M. A. Kumakhov, Phys. Lett. 57A, 17,1976, Dokl.Akad.SSSR,230,1077,1976

[5] M. A. Kumakhov, Zh.Eksp. Teor. Fiz. 72, 1489,1977, phys. stat. solidi (b), 1977,84-41, and phys. stat. solidi (b), 84, 581,1977

[6] X. Artru, S.P. Fomin, N.F. Shul'ga, K.A. Ispirian, N.K. Zhevago, Carbon nanotubes and fullerites in high-energy and X-ray physics, Phys. Rep. 412 (2005) 89-189.
[7] M. S. Dresselhaus, G. Dresselhaus and Ph. Avouris (Editors), Carbon Nanotubes: Synthesis, Structure, Properties and Applications (Springer, New York, 2001).

[8] V.V. Klimov, V.S. Letokhov, Monochromatic radiation emitted by a relativistic electron moving in a carbon nanotube, Phys. Lett. A 226 (1997) 244-252.

[9] G.V. Dedkov, Fullerene nanotubes can be used when transporting gamma quanta, neutrons, ion beams and radiation from relativistic particles, Nucl.Instr.Meth. B 143 (4) (1998) 584-590.

[10] M.K. Abu-Assy, M.S. Soliman , Nuclear Instruments and Methods in Physics Research B 384 (2016) 93-99.

[11] S. SATPATHY and A. P. PATHAK: Planar Channeling of Electrons and Posit,rons in Crystals, phys. stat. sol. (b) 153, 455 (1989).

[12] G. Moliere, Section A, Zeitschrift fur Naturforschung, Theorie der streuung schneller geladener Teilchen I. Einzelstreuung am abgeschirmten coulombfeld, J. Phys. Sci. 2 (3) (1947) 133-145 